\title{
High-Flow Nasal Cannula and Aerosolized $\beta$ Agonists for Rescue Therapy in Children With Bronchiolitis: A Case Series
}

\author{
Sherwin E Morgan RRT, Steve Mosakowski RRT, Patti Solano RRT, Jesse B Hall MD, and \\ Avery Tung MD
}

\begin{abstract}
Asthma and bronchiolitis are episodic obstructive pulmonary diseases characterized by bronchoconstriction, airway wall inflammation, increased mucus production, and air-flow obstruction. We present the cases of 5 infants treated for acute bronchiolitis with respiratory distress using a combination of high-flow nasal cannula oxygen (HFNC) and an Aerogen nebulizer to deliver aerosolized $\beta$-agonist therapy. In all infants, we found that HFNC resulted in a greater heart rate increase than delivery via a facemask. We also found that patients tolerated inhaled therapy better with HFNC than a facemask. Key words: asthma; bronchiolitis; $\beta$ agonists; HFNC; high-flow nasal cannula. [Respir Care 2015;60(9):e161-e165. (C) 2015 Daedalus Enterprises]
\end{abstract}

\section{Introduction}

Asthma and bronchiolitis are episodic obstructive pulmonary diseases characterized by bronchoconstriction, airway-wall inflammation, increased mucus production, and air-flow obstruction. ${ }^{1}$ Although both diseases result in significant morbidity and mortality, bronchiolitis is an increasing concern in small children, particularly when associated with new strains of viral infection such as respiratory syncytial virus, rhinovirus/enterovirus, human pneumovirus, and H1N1 virus. These infections range in severity from mild respiratory infections (common cold) to severe respiratory distress syndromes and can produce enough air-flow obstruction to cause respiratory failure. ${ }^{1,2}$ Clinically, both asthma and bronchiolitis result in the acute onset of respiratory distress, during which patients fre-

Mr Morgan, Mr Mosakowski, and Ms Solano are affiliated with the Department of Respiratory Care, Dr Hall is affiliated with the Section of Pulmonary and Critical Care Medicine, Department of Medicine, and Dr Tung is affiliated with the Department of Anesthesia and Critical Care, The University of Chicago, Chicago, Illinois.

Dr. Tung has disclosed a relationship with Anesthesia \& Analgesia. The other authors have disclosed no conflicts of interest.

Correspondence: Sherwin E Morgan RRT, Department of Respiratory Care, The University of Chicago, MC0981, 5841 South Maryland Avenue, Chicago, IL 60637. E-mail: sherwin.morgan@uchospitals.edu.

DOI: $10.4187 /$ respcare.03996 quently present to the clinic or pediatric emergency department for urgent care.

Current American Academy of Pediatrics guidelines do not recommend the use of bronchodilators for bronchiolitis. ${ }^{3}$ However, these guidelines are controversial, ${ }^{4}$ and because the initial differential diagnosis includes acute asthma attacks, clinicians may initially use jet nebulizers to deliver aerosolized $\beta$-agonist medications as a therapeutic trial. ${ }^{4}$ An inhaled $\beta$ agonist such as albuterol is often delivered using a jet nebulizer connected to a face mask. Use of a face mask, however, can often produce agitation, worsening respiratory distress and preventing effective drug delivery, particularly in young children. ${ }^{5}$

\section{Case Reports}

We present the cases of 5 infants treated in the pediatric emergency department at The University of Chicago Medicine Comer Children's Hospital. Two males 3 and 14 months of age and 3 females 2-21 months of age were brought to the pediatric emergency department seeking relief for moderate-to-severe respiratory distress. Initial clinical examination of all 5 children revealed wheezing, coughing, tachypnea, retractions, and decreased oxygen saturation $\left(\mathrm{S}_{\mathrm{pO}_{2}}<90 \%\right)$ on room air. The 5 patients had chest radiographs notable for atelectasis, pneumonia, and peribronchial thickening. Chest auscultation revealed rhonchi, wheezing, or diminished and coarse breath sounds. Upon presenting to the pediatric emergency department, all demonstrated a clinical asthma severity assessment score 
Table 1. Patient Ages, Disease Types, and Clinical Asthma Severity Assessment Scores Before and After Delivery of Aerosolized $\beta$ Agonist via Jet Nebulizer Connected to a Face Mask

\begin{tabular}{|c|c|c|c|c|c|c|}
\hline \multirow[b]{2}{*}{ Case } & \multirow[b]{2}{*}{ Sex } & \multirow[b]{2}{*}{ Age (mo) } & \multirow[b]{2}{*}{ Diseases } & \multicolumn{3}{|c|}{ Clinical Asthma Severity Assessment Scores } \\
\hline & & & & Initial & $\begin{array}{l}\text { After Jet Nebulizer/ } \\
\text { Face Mask Delivery }\end{array}$ & $\begin{array}{c}\text { After HFNC/ } \\
\text { Aerogen Solo Delivery }\end{array}$ \\
\hline 1 & Male & 3 & $\begin{array}{l}\text { Respiratory syncytial virus, } \\
\text { rhinovirus/enterovirus }\end{array}$ & 14 & 14 & 12 \\
\hline 2 & Female & 6 & Rhinovirus/enterovirus & 15 & 13 & 12 \\
\hline 3 & Female & 2 & Rhinovirus/enterovirus & 14 & 13 & 13 \\
\hline 4 & Male & 13 & Rhinovirus/enterovirus & 15 & 12 & 10 \\
\hline 5 & Female & 4 & Rhinovirus/enterovirus & 15 & 13 & 11 \\
\hline
\end{tabular}

consistent with moderate-to-severe exacerbations. This scoring system is a modified version of the Wood asthma scoring system, ${ }^{6}$ is age-specific, and includes assessment of breathing frequency, $\mathrm{S}_{\mathrm{pO}_{2}}$, auscultation, retraction, and dyspnea. Scores range from mild (5-7) or moderate (8-11) to severe (12-15). In all patients, initial treatment consisted of 2 consecutive doses of $0.083 \%$ albuterol delivered by a jet nebulizer connected to a face mask using $100 \%$ oxygen. Three patients also received racemic epinephrine $(2.25 \%)$ treatments delivered the same way and exhibited increased mucus production requiring frequent nasopharyngeal suctioning. Two patients had respiratory syncytial virus or rhinovirus/enterovirus, and 3 others had either rhinovirus or enterovirus. All patients had difficulty maintaining an $\mathrm{S}_{\mathrm{pO}_{2}}$ of $\geq 88 \%$ on room air and demonstrated only slight improvement with low-flow nasal cannula. After multiple albuterol treatments with the jet nebulizer connected to a face mask, clinical asthma severity assessment scores in all patients remained in the moderateto-severe range (Table 1).

Due to persistent air-flow obstruction and increasing concern for fatigue and respiratory failure, the pediatric emergency department staff considered more aggressive respiratory support options, including nasal CPAP, bi-level positive airway pressure, heliox (helium-oxygen mixture), or intubation. Because infants with bronchiolitis and obstructive lung disease can be difficult to manage on positive-pressure ventilation, high-flow nasal cannula (HFNC) was attempted because it reduces the risk of intubation in infants with respiratory failure. ${ }^{7}$ HFNC was implemented at oxygen gas flows set between 5 and $8 \mathrm{~L} / \mathrm{min}$ with a target $\mathrm{F}_{\mathrm{IO}_{2}}$ of 1.0 .

To avoid diluting the $\mathrm{F}_{\mathrm{IO}_{2}}$ with HFNC use, the gas flow must meet or exceed the inspiratory flow demands of the patient. We set the $\mathrm{O}_{2}$ flow by estimating the expired tidal volume for each patient and multiplying by the breathing frequency to obtain the estimated expired minute volume. We then set the oxygen gas flow to $\sim 3.5$ times higher the expired minute volume and/or 5-8 L/min based on the manufacturer's instructions for the nasal cannula.
After HFNC implementation, $\mathrm{S}_{\mathrm{pO}_{2}}$ rose to $\geq 95 \%$ at $100 \% \mathrm{~F}_{\mathrm{IO}_{2}}$, but average clinical asthma severity assessment scores remained unchanged $(14.6 \pm 0.55$ before HFNC application and $13 \pm 0.71$ after). We thus added aerosolized $\beta$-agonist delivery via a jet nebulizer connected to a face mask to the HFNC treatment. All patients thus received $0.083 \%$ albuterol and aerosolized steroid treatments via the jet nebulizer and face mask every $2 \mathrm{~h}$ along with HFNC and chest physiotherapy. However, all patients remained agitated and combative.

To reduce anxiety, agitation, and work of breathing, we then removed the jet nebulizer and face mask and delivered inhaled $\beta$ agonist via a nebulizer connected to the HFNC alone. Oxygen was administered via an RT329 circuit (Fisher \& Paykel Healthcare, Auckland, New Zealand). The gas inlet valve was connected to the dry side of a Fisher \& Paykel MR290 humidifier chamber with oxygen tubing to the gas inlet adapter which was connected to an oxygen flow meter via a nipple adapter (Fig. 1). The humidifier chamber was then connected to the HFNC. The temperature control was set to the noninvasive mode. We used a mesh nebulizer (Aerogen Solo, Aerogen, Galway, Ireland) to deliver aerosolized medicine. The delivery system consisted of the Aerogen Solo connected on either the dry or wet side of the MR290 humidifier chamber. Albuterol $(3 \mathrm{~mL})$ was then placed into the Aerogen Solo medication chamber. The Aerogen Solo control unit was powered on, and visual verification of nebulized medication delivery was performed at 2 points in the RT329 circuit.

To assess clinical effect, we monitored heart rates and clinical asthma severity assessment scores before, during, and after each treatment via the jet nebulizer and face mask and via HFNC and the Aerogen Solo. We found that scores were unchanged after delivery via HFNC and the Aerogen Solo (see Table 1), but that heart rates increased to a greater degree when albuterol was delivered via HFNC and the Aerogen Solo compared with the jet nebulizer and face mask (Table 2). 


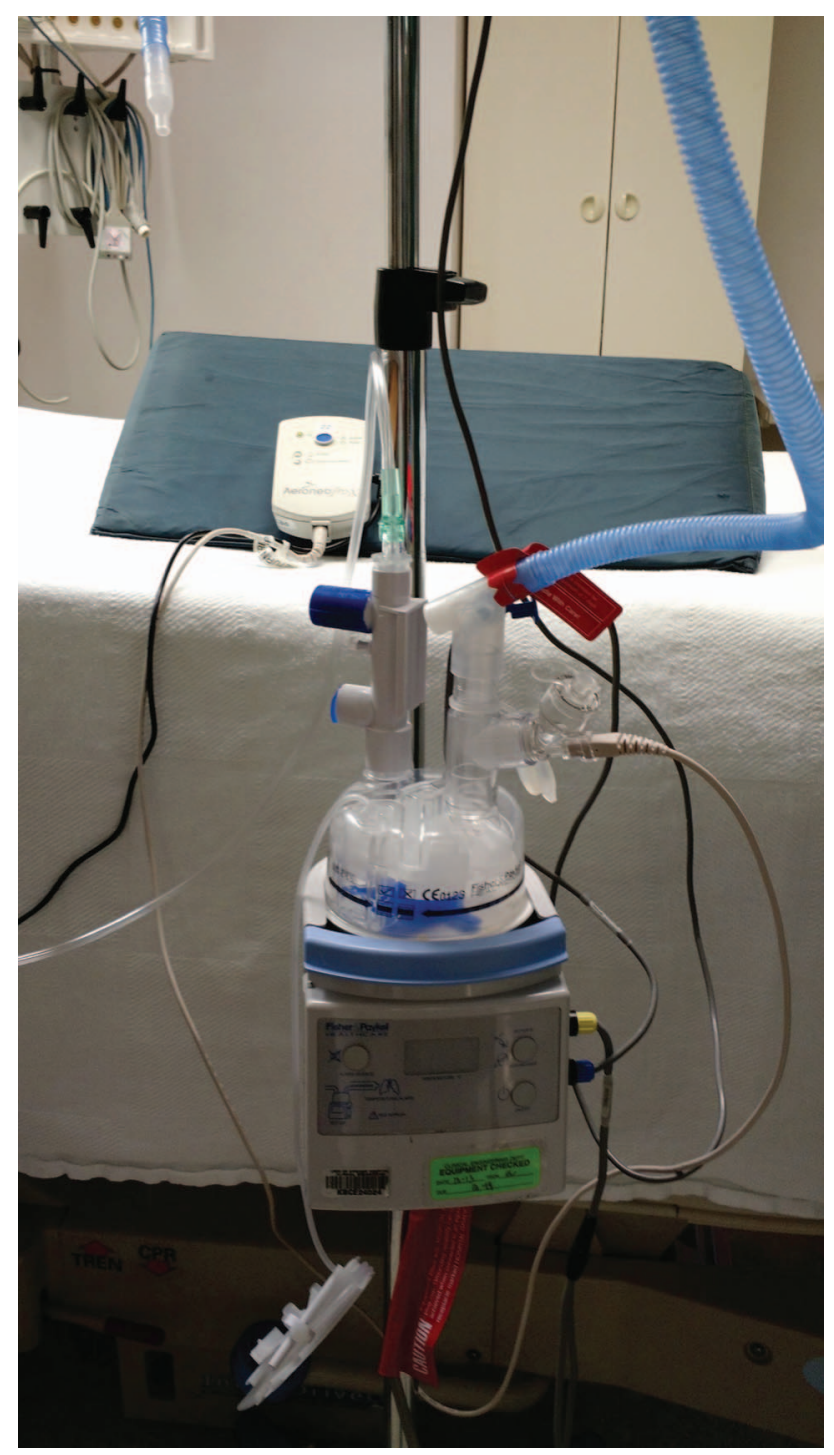

Fig. 1. Apparatus for delivering nebulized drug using high-flow nasal cannula and the Aerogen Solo. The nebulizer is located on the wet (right) side of the MR850 humidifier, and the gas inlet with a pressure safety valve is located on the dry (left) side.

More importantly, we found that patient agitation was markedly improved when albuterol was delivered via HFNC and the Aerogen Solo alone. During treatment with the jet nebulizer connected to a face mask with or without HFNC, all 5 patients were increasingly agitated due to the face mask, and as a result, clinical asthma severity assessment scores in all patients increased from moderate to severe. In addition, during treatment, the parent would frequently have to hold the patient in an attempt to keep the face mask on the patient's face. In contrast, when $\beta$ agonist was delivered via HFNC and the Aerogen Solo alone, patients were calm enough to sleep through their treatment while being observed closely by respiratory therapists, nurses, and parents. Evidence that $\beta$-agonist deliv- ery was effective was also indirectly provided by heart rate (see Table 2). We found increases in heart rate with HFNC and the Aerogen Solo, but no changes in any other cardiorespiratory parameter, including mean arterial blood pressure, breathing frequency, and $\mathrm{S}_{\mathrm{pO}_{2}}$. Comfort and anxiety levels for both patients and parents were considerably improved with treatment via HFNC and the Aerogen Solo. Two patients previously in respiratory distress while using the jet nebulizer and face mask were able to breastfeed while receiving bronchodilator therapy via HFNC and the Aerogen Solo, and 3 others were able to use pacifiers during treatment via HFNC and the Aerogen Solo. All patients clearly preferred HFNC/Aerogen Solo delivery to jet nebulizer/face mask delivery.

\section{Discussion}

Although HFNC is increasingly used as a support strategy for infants with respiratory distress, data on its effectiveness are mixed. ${ }^{1}$ Lee et $\mathrm{al}^{7}$ reported that $\mathrm{HFNC}$ is a useful respiratory support strategy for bronchiolitis. In contrast, a 2011 Cochrane review found that HFNC may be associated with a higher rate of re-intubation than nasal CPAP in preterm infants, ${ }^{8}$ and the 2014 update concluded that no evidence was available to assess safety or effectiveness. ${ }^{9}$ Although current pediatric guidelines do not recommend the use of inhaled $\beta$ agonists for acute bronchiolitis, ${ }^{3}$ inhaled $\beta$-agonist treatment in this patient population may still be relevant, as the differential diagnosis for bronchiolitis includes acute asthma. ${ }^{4}$

Several challenges complicate the delivery of inhaled drugs in infants with acute respiratory distress from severe bronchiolitis. Children are mainly nasal breathers, ${ }^{10}$ and inhalation through the nostrils is common when using the a jet nebulizer connected to a face mask. ${ }^{5,10}$ In adult patients, the nasal passage filters out $35-50 \%$ of the aerosol, ${ }^{11}$ and such filtering may also affect medication delivery in children. In addition, young children may become agitated when a face mask is placed over their mouth and nose. ${ }^{5}$ Other factors that may affect medication delivery are the presence of a pacifier in the mouth and increased anxiety and stress from parents seeing their children in distress. It is thus reasonable that patients may become agitated and combative when treated with aggressive aerosol therapy via a jet nebulizer and face mask. Moreover, although oxygen saturation may improve with this combination, it may be the result of powering with $100 \%$ oxygen, and clinical asthma severity assessment scores themselves may not change. One study indicated that $47 \%$ of children did not tolerate a jet nebulizer connected to a face mask. ${ }^{10}$

Existing evidence suggests that inhaled medication delivery using a face mask is poor. Amirav et al ${ }^{12}$ used gamma scintigraphy to assess the pulmonary distribution 
Table 2. Effect of Aerosolized $\beta$-Agonist Delivered via a Jet Nebulizer and Face Mask and via HFNC and the Aerogen Solo on Heart Rate

\begin{tabular}{|c|c|c|c|c|c|}
\hline \multirow{2}{*}{ Case } & \multirow{2}{*}{ Age (mo) } & \multicolumn{2}{|c|}{$\begin{array}{l}\text { Jet Nebulizer/Face Mask Delivery } \\
\text { (beats/min) }\end{array}$} & \multicolumn{2}{|c|}{$\begin{array}{l}\text { HFNC/Aerogen Solo Delivery } \\
\text { (beats/min) }\end{array}$} \\
\hline & & Before & After & Before & After \\
\hline 1 & 3 & 121 & 125 & 123 & 178 \\
\hline 2 & 6 & 133 & 140 & 130 & 188 \\
\hline 3 & 2 & 129 & 136 & 132 & 194 \\
\hline 4 & 13 & 141 & 149 & 143 & 190 \\
\hline 5 & 4 & 138 & 142 & 140 & 186 \\
\hline Mean \pm SD & & $132.4 \pm 7.8$ & $138.4 \pm 8.8$ & $133.6 \pm 8.0$ & $187.2 \pm 5.9$ \\
\hline
\end{tabular}

$\overline{\mathrm{HFNC}}=$ high-flow nasal cannula

of technetium-99m albuterol aerosol in 12 infants with acute bronchiolitis. They found that only $1.5 \%$ reached the lung, $7.8 \%$ was deposited in the upper respiratory and gastrointestinal tracts, and 10-12\% remained on the subject's face. The authors concluded that poor aerosol deposition was common when face mask techniques were used. Ditcham et al ${ }^{13}$ administered radiolabeled salbutamol via a spacer or nebulizer and face mask to 8 infants and found that overall mask deposition was $0.8-5.2 \%$ and fell to $0.6-0.4 \%$ when children were agitated or screaming. Clay et al ${ }^{14}$ found that changing drug concentration had little effect on aerosol size or drug mass median diameter during treatment, but that aerosol particle size remained at $<1.3 \mu \mathrm{m}$ when the optimal breathing dose range was $<5 \mu \mathrm{m}$ regardless of drug concentration. Taken together, these data suggest that improved methods for delivering inhaled medications are needed.

Although we did not use an objective agitation scale, we (and nurses caring for these patients) clearly found decreased agitation and heart rate evident of adequate inhaled $\beta$-agonist delivery when aerosolized albuterol was delivered by HFNC and the Aerogen Solo. Our patients were less agitated, and their heart rates increased to a greater degree with HFNC and the Aerogen Solo than with the jet nebulizer and face mask. Although this effect may have been due to agitation resulting from the jet nebulizer/face mask combination itself, we have not found our other patients to be agitated, so we believe that this possibility is unlikely. Our observations support a strategy of delivering inhaled albuterol via HFNC and the Aerogen Solo rather than a jet nebulizer connected to a face mask.

Existing in vitro simulation data support using a nasal cannula for aerosol delivery. ${ }^{15}$ Our approach is noteworthy not only because of the nasal cannula delivery route but also because of the nebulization method we used. Unlike a jet nebulizer, which uses the gas source to function and limits the flows that can be used, a vibrating mesh nebulizer does not require a gas source to function and allows a wider range of gas flows. Moreover, a vibrating mesh nebulizer generates smaller particle sizes than a jet nebulizer, ${ }^{16}$ which may facilitate medication delivery.

The use of HFNC and the Aerogen Solo to deliver aerosolized medications has been investigated in lung models. These studies have had mixed results, in part because 2 different HFNC systems were used. In one study, the investigators used the Vapotherm HFNC system (Stevensville, MD) rather than the Fisher \& Paykel system used in our case series and found that $80 \%$ of the aerosolized medication was trapped in the nasal cannula apparatus. ${ }^{17}$ They suggested that cannula size and gas flows influenced aerosol delivery. Another potential reason is that the nebulizer in the Vapotherm system is located after the humidifier in the delivery circuit, which can result in high humidity rainout of nebulized medication. In another in vitro study using the Aerogen system, the nebulizer apparatus was placed just before the humidification chamber. ${ }^{16}$ That authors demonstrated that the Aerogen nebulizer functioned better when located before rather than after the humidification chamber, but that delivery of aerosolized medication was poor at gas flows of $\leq 3 \mathrm{~L} / \mathrm{min}$. Another in vitro study demonstrated that $18-26 \%$ of the medication dose was delivered using a pediatric nasal cannula, and the authors concluded that aerosolized medications can be effectively delivered via HFNC. ${ }^{18}$

In conclusion, although many in vitro studies exist, few clinical reports of aerosolized bronchodilator administration via HFNC and the Aerogen Solo exist. We report 5 cases of infants with bronchiolitis who were given $\beta$-agonist bronchodilators via HFNC and the Aerogen Solo. Although clinical asthma severity assessment scores did not appreciably change with HFNC/Aerogen Solo therapy, and recent guideline changes now recommend against $\beta$ agonists in bronchiolitis patients, we believe that the combination of HFNC and the Aerogen Solo was clinically better tolerated in our patients, possibly preventing them from escalating to more invasive respiratory support. We hypothesize that the heart rate increase we observed with the HFNC/Aerogen Solo delivery option represented clin- 


\section{Aerosol Delivery During HFNC in Bronchiolitis}

ically relevant medication delivery and absorption and verified aerosolized medication output by disconnecting the RT329 circuit from the nasal cannula to observe aerosolized mist exiting the oral cavity on exhalation. More prospective clinical studies are needed to further define how this population of patients may benefit from HFNC/Aerogen Solo use.

\section{REFERENCES}

1. McKiernan C, Chua LC, Visintainer PF, Allen H. High flow nasal cannulae therapy in infants with bronchiolitis. J Pediatr 2010;156(4): 634-638.

2. Morgan SE, Vukin K, Mosakowski S, Solano P, Stanton L, Lester L, et al. Use of heliox delivered via high-flow nasal cannula to treat an infant with coronavirus-related respiratory infection and severe acute air-flow obstruction. Respir Care 2014;59(11):e166-e170.

3. Ralston SL, Lieberthal AS, Meissner HC, Alverson BK, Baley JE, Gadomski AM, et al. Clinical practice guideline: the diagnosis, management, and prevention of bronchiolitis. Pediatrics 2014;134(5): e1474-502.

4. Walsh P, Rothenberg SJ. American Academy of Pediatrics 2014 bronchiolitis guidelines: bonfire of the evidence. West J Emerg Med 2015;16(1):85-88.

5. Cole CH. Special problems in aerosol delivery: neonatal and pediatric considerations. Respir Care 2000;45(6):646-651.

6. Wood DW, Downes JJ, Lecks HI. A clinical scoring system for the diagnosis of respiratory failure. Preliminary report on childhood status asthmaticus. Am J Dis Child 1972;123(3):227-228.

7. Lee JH, Rehder KJ, Williford L, Cheifetz IM, Turner DA. Use of HFNC in critically ill infants, children, and adults: a critical review of literature. Intensive Care Med 2013;39(2):247-257.
8. Wilkinson D, Andersen C, O'Donnell CP, De Paoli AG. High flow nasal cannula for respiratory support in preterm infants. Cochrane Database Syst Rev 2011;5:CD006405.

9. Mayfield S, Jauncey-Cooke J, Hough JL, Schibler A, Gibbons K, Bogossian F. High-flow nasal cannula therapy for respiratory support in children. Cochrane Database Syst Rev 2014;3:CD009850.

10. Coates AL, Ho SL. Drug administration by jet nebulization. Pediatr Pulmonol 1998;26(6):412-423.

11. Rubin BK. Pediatric aerosol therapy: new devices and new drugs. Respir Care 2011;56(9):1411-1421; discussion 1421-1423.

12. Amirav I, Balanov I, Gorenberg M, Luder AS, Newhouse MT, Groshar D. Beta-agonist aerosol distribution in respiratory syncytial virus bronchiolitis in infants. J Nucl Med 2002;43(4):487-491.

13. Ditcham W, Murdzoska J, Zhang G, Roller C, von Hollen D, Nikander K, Devadason SG. Lung deposition of ${ }^{99 \mathrm{~m}}$ Tc-radiolabeled albuterol delivered through a pressurized metered dose inhaler and spacer with facemask or mouthpiece in children with asthma. J Aerosol Med Pulm Drug Deliv 2014;27(Suppl 1):S63-S75.

14. Clay MM, Pavia D, Newman SP, Clarke SW. Factors influencing the size distribution of aerosols from jet nebulisers. Thorax 1983;38(10): 755-759.

15. Amirav I, Borojeni AA, Halamish A, Newhouse MT, Golshahi L. Nasal versus oral aerosol delivery to the "lungs" in infants and toddlers. Pediatr Pulmonol 2015;50(3):276-283.

16. Ari A, Atalay OT, Harwood R, Sheard MM, Aljamhan EA, Fink JB. Influence of nebulizer type, and bias flow on aerosol drug delivery in a simulated pediatric and adult lung model during mechanical ventilation. Respir Care 2010;55(7):845-851.

17. Perry SA, Kesser KC, Geller DE, Selhorst DM, Rendle JK, Hertzog $\mathrm{JH}$. Influences of cannula size and flow rate on aerosol drug delivery through the Vapotherm humidified high flow nasal cannula system. Pediatr Crit Care Med 2013;14(5):e250-e256.

18. Bhashyam AR, Wolf MT, Marcinkowski AL, Saville A, Thomas K, Carcillo JA, Corcoran TE. Aerosol delivery through nasal cannulas: as in vitro study. J Aerosol Med Pulm Drug Deliv 2008;21(2):181-188. 